\title{
The Functional Connectivity between the Locust Leg Pattern Generating Networks and the Subesophageal Ganglion Higher Motor Center
}

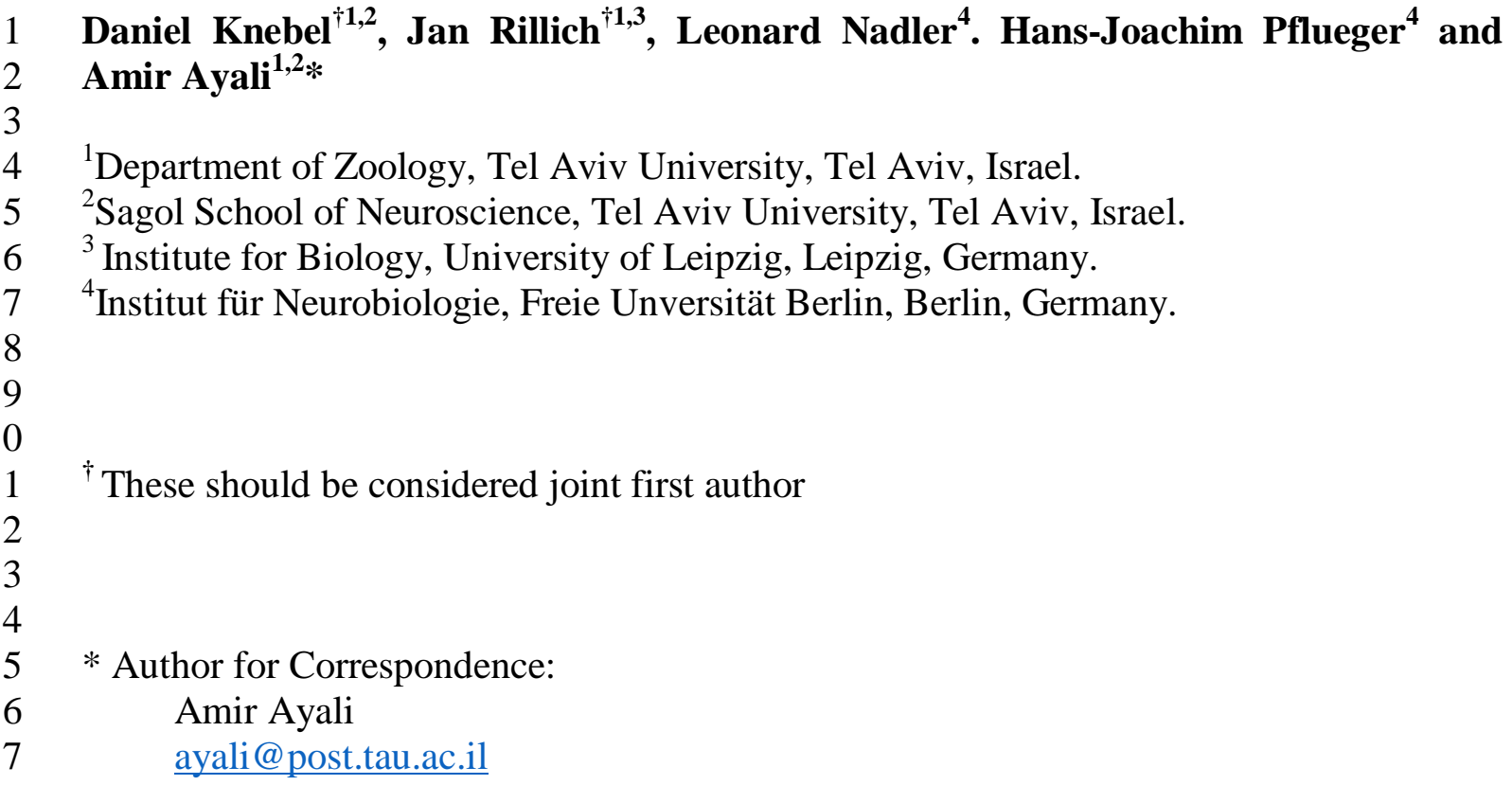

Acknowledgements

20 This work was partially supported by DAAD travel scholarships (to DK) and, in its final

21 stages, by the German Research Council (DFG; Grant RI 2728/2-1). H-J P gratefully 22 acknowledges the support by the DFG (FOR 1363, Pf128/30-1 and PF128/32-1) and the 23 receipt of a Berlin NaFoeG-stipend to $\mathrm{LN}$. We thank Baruch Barzel for his valuable 24 support in the data analysis.

28 The authors declare no conflict of interests 


\section{Abstract}

31 Interactions among different neuronal circuits are essential for adaptable

32 coordinated behavior. Specifically, higher motor centers and central pattern generators

33 (CPGs) induce rhythmic leg movements that act in concert in the control of locomotion.

34 Here we explored the relations between the subesophageal ganglion (SEG) and thoracic

35 leg CPGs in the desert locust. Backfill staining revealed about 300 SEG descending

36 interneurons (DINs) and some overlap with the arborization of DINs and leg motor

37 neurons. In accordance, in in-vitro preparations, electrical stimulation applied to the SEG

38 excited these neurons, and in some cases also induced CPGs activity. Additionally, we

39 found that the SEG regulates the coupling pattern among the CPGs: when the CPGs were

40 activated pharmacologically, inputs from the SEG were able to synchronize contralateral

41 CPGs. This motor output was correlated to the firing of SEG descending and local

42 interneurons. Altogether, these findings point to a role of the SEG in both activating leg

43 CPGs and in coordinating their oscillations, and suggest parallels between the SEG and

44 the brainstem of vertebrates.

47 Keywords: locomotion, locust, insect, subesophageal ganglion, central pattern generator

48 (CPG), intersegmental coordination 


\section{Introduction}

51 A longstanding and fundamental question in neuroscience is that of how the brain

52 coordinates motor behaviors (Penfield and Boldrey, 1937; Schmidt and Lee, 2005;

53 Fritsch and Hitzig, 2009). Ample research across species and methodologies has revealed

54 a differentiation between three levels of motor control: central pattern generators (CPGs -

55 neuronal oscillators that can produce rhythmic motor output in the absence of sensory or

56 descending inputs; see recent reviews in Marder and Bucher, 2007; Mulloney and

57 Smarandache, 2010; Marder, 2012; Rybak et al., 2015); sensory feedback; and higher

58 motor centers. In most cases, the CPGs determine rhythmic alternating activity of specific

59 antagonistic muscles. The CPGs by themselves, or often together with a few of the many

60 sensory feedback loops in intact animals, are sufficient for the generation of coordinated

61 motor patterns (e.g. Bal et al., 1988; Stevenson and Kutsch, 1987). Higher motor centers,

62 on the other hand, have a wide-ranging influence, affecting various body parts

63 concurrently. They may select the appropriate behavior, initiate it, and orchestrate it. To

64 this end, higher motor centers form functional connections with CPGs and may modulate

65 different ones simultaneously. This report describes the interplay between leg CPGs and

66 subesophageal ganglion (SEG), which serves as a higher motor center for locomotion

67 (Gal and Libersat, 2006; Kaiser and Libersat, 2015; Tastekin et al., 2015), of the desert

68 locust, Schistocerca gregaria (Forskål).

69 A comprehensive body of work describing the interactions between higher motor

70 centers and CPGs in the tadpole largely inspired our current work, which shares similar

71 aims with it. Each of the tadpole's spinal cord segments includes two CPGs that produce

72 an alternating rhythmic motor output, activating the myotomal muscles and thus inducing

73 stereotypic swimming movements (Kahn and Roberts, 1982a). These segmental circuits

74 reciprocally inhibit each other, while maintaining a phase difference between the activity

75 of consecutive segments, thus producing the longitudinal directionality of the movement

76 (Kahn and Roberts, 1982a; b). However, when the spinal cord is isolated from the

77 hindbrain, only short fictive swim bouts can be generated (Li et al., 2006). Further

78 research into these findings revealed that in addition to the spinal network of CPGs,

79 another rhythm-generating center resides in the hindbrain, consisting of electrically

80 coupled descending interneurons (DINs), which are active before each cycle of the spinal 
81 motor output, and drive the spinal CPGs (Li et al., 2006, 2009, 2010; Soffe et al., 2009).

82 Similarly, studies in mice and lampreys have pointed to an instrumental role of the brain

83 stem in locomotion (McClellan and Grillner, 1984; Dubuc et al., 2008; Gordon and

84 Whelan, 2008; Hägglund et al., 2010).

85 To date, the study of higher locomotion centers in insects has mostly focused on 86 their behavioral role in-vivo. Using lesions, genetic techniques, and electrophysiological

87 recordings, certain areas of the insect supraesophageal ganglion (brain), such as the

88 central complex and mushroom bodies, were shown to control advanced aspects of 89 walking: for example, speed change and turning (e.g. Strauss, 2002; Gal and Libersat,

90 2006; Poeck et al., 2008; Bender et al., 2010; Guo and Ritzmann, 2013). The SEG, which 91 anatomically resides between the brain and the thoracic ganglia, was considered 92 responsible for more basic features of walking such as initiation, maintenance, and 93 forward-backward orientation (Huber, 1960; Kien and Altman, 1984; Bässler et al., 1985;

94 Kien, 1990a; Gal and Libersat, 2008; Bidaye et al., 2014). In accordance, lesion-based 95 experiments have demonstrated that removal of the brain does not abolish spontaneous 96 walking, whereas the removal of the SEG eliminates it (Kien and Williams, 1983; Gal 97 and Libersat, 2006). Our knowledge of the anatomical and moreover functional 98 connections between the SEG and leg CPGs is, however, still incomplete.

99 In accord with previous studies that found central connections between the locust

100 limbs (e.g. Berkowitz and Laurent, 1996), we have recently offered a detailed description 101 of the functional connections among the inter-leg coxa-trochanteral CPGs in the locust 102 in-vitro (Knebel et al., 2017). We showed that each of the three thoracic ganglia has its 103 own default, inherent, bilateral coupling of these CPGs: in-phase within the pro- and 104 mesothoracic ganglia, and anti-phase in the metathoracic ganglion. Furthermore, each 105 ganglion was found to be capable of imparting its coupling scheme onto the other 106 ganglia. Importantly, as in most walking systems (Büschges et al., 2011), none of the 107 observed in-vitro inter-leg coordination schemes resembled a functional walking gait, and 108 specifically not the tripod gait common among insects, in which neighboring pairs of legs 109 show antiphase activity (Wilson, 1966; Grabowska et al., 2012). The thoracic network of 110 oscillators must, therefore, feature inherent flexibility in order to establish a functional 111 walking gait. 
112 In the current report we follow-up on this work, taking advantage of the locust's

113 easily accessible nervous systems, as well as its anatomical modularity, i.e. the functional

114 and anatomical separation of the leg CPGs, compartmented by the three interconnected

115 segmental thoracic ganglia; and the higher locomotion centers, encompassed in the two

116 head ganglia - the brain and SEG (or gnathal ganglia; Ito et al., 2014). We introduce first

117 evidence of leverage points for central regulation of the leg CPG network by the SEG

118 higher motor center.

119 Materials and Methods

$120 \quad$ Experimental animals

121 All experiments were performed on adult male desert locusts (Schistocerca

122 gregaria) from our colony at Tel Aviv University (Ayali and Zilberstein, 2002), within

123 the first two weeks after the final molt. All experiments complied with the Principles of

124 Laboratory Animal Care and the Israeli Law regarding the protection of animals.

125 Preparation

126 Recordings were conducted from in-vitro ventral nerve chord preparations, 127 including the pro-, meso- and metathoracic ganglia, and the SEG. Prior to dissection, the 128 animals were anesthetized with $\mathrm{CO}_{2}$ for at least $5 \mathrm{~min}$. Following removal of the brain 129 (decerebration), appendages, the pronotal shield, and the abdomen posteriorly to the 130 fourth abdominal segment, a longitudinal cut was performed in the cuticle along the 131 dorsal midline of the thorax. The preparation was pinned to a Sylgard dish (Sylgard 182 132 silicon Elastomer, Dow Corning Corp.), and the cut was widened and superfused with 133 locust saline containing (in $\mathrm{mM}$ ): $150 \mathrm{NaCl}, 5 \mathrm{KCl}, 5 \mathrm{CaCl}_{2}, 2 \mathrm{MgCl}_{2}, 10 \mathrm{Hepes}_{2} 25$ 134 sucrose at $\mathrm{pH}$ 7.4. Air sacs and fatty tissue covering the ventral nerve cord were removed, 135 and the thoracic ganglia chain and SEG with their surrounding tracheal supply were 136 dissected out of the animal body, pinned in a clean Sylgard dish, dorsal side up, and 137 bathed in locust saline. The two main tracheae were opened and floated on the saline 138 surface. All peripheral nerve branches originating from the thoracic ganglia were cut 139 short except for the N5a nerves (numbered after Campbell, 1961), which each contain 
140 three motor axons: the slow and fast trochanteral depressors and a common inhibitor (Fig.

$141 \quad 1$; Ds, Df, and CI respectively).

\section{Electrophysiology and neuroanatomy}

143 To test the effect of SEG electrical stimulation on the thoracic motor output, trains 144 of short electrical pulses (200 ms of $250 \mathrm{~Hz}$ pulses of $0.5 \mathrm{~ms}$ each, $1 \mathrm{~V}$ ) were delivered to 145 different areas of the SEG by carefully-fashioned insulated platinum-iridium electrodes, 146 with especially high resistance and small tip diameter $(15 \mu \mathrm{m})$, as described by Hussaini 147 and Menzel (2013). Thus, we limited the stimulated area as much as possible. All 148 electrical stimulations were generated by a Master 8 stimulator (A.M.P.I).

149 The activity of the motor nerves (N5a) of each thoracic ganglion was extracellularly 150 recorded by suction electrodes made of borosilicate glass capillaries (A-M systems) and 151 pulled with a P87 puller (Sutter Instruments). Unipolar hook electrodes were used to 152 monitor inter-segmental information transfer from the inter-ganglia connectives (SEG153 pro, pro-meso, meso-meta). Data were acquired using two four-channel differential AC 154 amplifiers (Model 1700, A-M Systems).

155 For intracellular recordings, the SEG neurons were impaled with borosilicate glass 156 capillaries pulled with the P87 puller. The electrode tips were filled with $3 \%$ Neurobiotin 157 (Vector Laboratories, Inc.) diluted in 3M potassium acetate and the shafts with $1583 \mathrm{M}$ potassium acetate alone, leaving a small air bubble in between. These electrodes had 159 a resistance of approximately $60 \mathrm{M} \Omega$. In order to penetrate the ganglion sheath, a few 160 crystals of protease (Sigma-Aldrich) were placed on the ganglion for $30 \mathrm{~s}$. Intracellularly 161 recorded signals were amplified using a DC amplifier (Axoclamp-2B, Molecular 162 Devices). All recordings were digitized (Axon Digidata 1440A A-D board) and stored on 163 a computer using standard software (Axo-Scope software; Molecular Devices). Resting 164 membrane potentials for all intracellular recordings shown were below $-65 \mathrm{mV}$. After 165 evaluating the responses to restricted drug application, the intracellularly recorded neuron 166 was labeled by iontophoretic injection of Neurobiotin using depolarizing current pulses 167 (2 nA; $100 \mathrm{~ms} ; 5 \mathrm{~Hz} ; 20-40 \mathrm{~min})$.

168 Backfill staining was accomplished by cutting the desired nerve and bathing it in 169 Dextran-Rhodamine (Molecular probes) or Neurobiotin. After intracellular labeling or 
170 backfill staining, preparations were placed for $24 \mathrm{~h}$ in a moisture chamber at $4^{\circ} \mathrm{C}$ to allow

171 the tracer to diffuse along the neurons. Thereafter, the nerve chord was fixed for $2-4 \mathrm{~h}$ in

$1723 \%$ paraformaldehyde. The ganglia sheaths were permeabilized with a $0.1 \%$

173 collagenase/dispase (Sigma-Aldrich) solution for $30 \mathrm{~min}$ at $36^{\circ} \mathrm{C}$ before the Neurobiotin

174 labeling was visualized by streptavidin-Cy3 (Jackson ImmunoResearch Labs). Finally,

175 the ganglia chain was dehydrated in an ethanol series and cleared in methyl-salicylate

176 (Merck KGaA). The whole-mount preparations were scanned with a confocal microscope

177 (either ZEISS LSM 510, Carl Zeiss, or LEICA TCS SP2, Leica). Neurons were 178 reconstructed from confocal image stacks using Fiji software 179 (http://fiji.sc/wiki/index.php/Fiji).

\section{Pharmacological treatment}

The muscarinic receptor agonist pilocarpine hydrochloride (Sigma-Aldrich, St

182 Louis, MO, USA) was dissolved in locust saline to a final concentration of $0.5 \mathrm{mM}$, 183 which typically elicits rhythmic motor activity in leg motor nerves (e.g. Ryckebusch and 184 Laurent, 1993). After $5 \mathrm{~min}$ in normal saline, the pilocarpine solution was restrictively 185 bath-applied to the metathoracic ganglion, which was isolated from the rest of the ventral 186 nerve cord by surrounding it with a petroleum jelly (Vaseline) wall. All other thoracic 187 ganglia and the SEG were bathed in normal saline. The well was carefully leak proofed 188 by applying saline, first only to the surrounding of the well, then into the well only, and checking for leaks from the well walls.

\section{Data analysis}

The pilocarpine-induced motor activity in the thoracic ganglia was measured in 31

192 experiments, initially with the SEG intact and subsequently again after disconnecting the

193 SEG. In each experiment between three to seven simultaneous recordings were conducted

194 from the leg depressor motor neuron (MN) pools, including the slow and fast trochanteral

195 depressors and common inhibitor (Fig. 1; Ds, Df, and CI respectively). Spikes were

196 detected and identified based on their amplitude, and only activity of the excitatory MNs

197 was taken into account, without separating between the Ds and Df. Additionally, in some

198 of the experiments the activity of the inter-ganglia connectives and SEG interneurons was 
199 also monitored. We routinely evaluated the activity in two subsequent 8 min windows:

200 the first, typically after 22 min post drug application, and the second immediately after

201 the neck connectives were cut.

202 To identify SEG DINs in the connective recordings, spikes were first identified 203 using a template recognition function (Dataview software, University of St. Andrews) in 204 one of the channels. Subsequently, we overlaid all identified spikes in $20 \mathrm{~ms}$ windows by 205 aligning their maximum point. We repeated this process for the parallel time windows in 206 the other connectives recordings, and averaged each of the overlays. This allowed us to 207 examine the typical activity before and after the identified spike in the more rostral and/or 208 caudal connective recordings. Based on a typical axonal conduction speed of $2 \mathrm{~m} / \mathrm{s}$ 209 (Gray and Robertson, 1998), and a distance of 3-5 mm between each pair of electrodes, 210 we expected DINs spikes to appear at a delay of about 1.5-2.5 ms between electrodes 211 monitoring adjacent ipsilateral connectives, from rostral to caudal. Spikes that were not 212 accompanied by such preceding or delayed activity in adjacent connectives were filtered 213 out. In one experiment, we used hook electrodes to record both neck connectives and to 214 identify simultaneous bilateral descending spikes. After verifying that the spike was 215 descending (as described above), we selected only simultaneous spikes in both 216 connectives.

217 To characterize the phases between the output of pairs of CPGs, we used cross218 spectrum analysis in MATLAB (MathWorks Inc.), following a procedure developed by 219 Miller and Sigvardt, (1998; see also Sigvardt and Miller, 1998). Only significantly 220 coherent frequencies of each pair of recordings were used to calculate each experiment 221 mean phase vector. The Watson-Williams F-test was used to test for differences in the 222 phase vectors. In order to combine both the uniformity and directionality of the phase 223 distributions, we used the synchronization index, ranging from -1, perfect anti phase, to $224+1$, perfect in phase. This was calculated by averaging all the experiments mean phase 225 vectors, and projecting the product on the 0-180 axis (see Knebel et al., 2017 for more 226 details on the cross-spectrum and synchronization index).

227 In order to compare the SEG neuronal output and the activity of thoracic MN pools, 228 we utilized cross-covariance analysis in MATLAB. We used a smoothed on-time vector 229 of the spikes of each of the simultaneous recordings. It should be noted that the resultant 
230 covariance coefficient value was normalized in order to be able to compare between

231 different analyses. The coefficient is valid only relatively as it is highly dependent on the

232 prior smoothing process: the change of the coefficient over time represents the relative

233 likelihood of spikes to occur in different temporal circumstances, and can be compared

234 among the different examples presented that went through the exact same process.

235 We tested the statistical significance of the obtained covariance coefficients by 236 performing the exact same analysis on 1000 randomly chosen pairs of the motor output 237 recordings, in which each recording came from a different experiment (different locust).

238 Thereby, we could calculate a bootstrap which represented a confidence interval of $95 \%$,

239 which defined the range of covariance coefficients that could be obtained by chance $240(<95 \%)$. The extreme values of the bootstrap were 1.11 and -0.968 . Thus, any result that 241 crosses these have less than a 5\% chance of being a type 1 error, and therefore can be 242 considered statistically significant. All the results which are mentioned as meaningful 243 crossed this threshold, unless noted otherwise.

244 To merge the cross-covariance of the activity of two MN pools and SEG neuronal 245 activity, we multiplied the two normalized cross-covariances calculated separately for 246 each MN pool against the SEG neuronal activity. This yielded a square matrix, in which 247 the axes represent the lags of each cross-covariance. Thus, the diagonal axis (from 248 bottom left to top right) depicts the on-time correlation of the $\mathrm{MN}$ pool in respect to the 249 SEG activity, while any deviation from this line indicates the correlation value at a 250 certain time-lag. Again, the absolute values of this analysis are relative, but can be 251 compared among themselves to show the tendency of firing at different temporal states of 252 the network.

\section{Results}

254 All electrophysiological experiments $(\mathrm{N}=40)$ were performed on isolated ventral 255 nerve cords of the locust, including the SEG and the three thoracic ganglia. In all, we 256 evaluated the activity of the coxa-trochanteral CPGs, which induce alternated motor 257 output between the leg levator and depressor motor neuron pools (Ryckebusch and 258 Laurent, 1993; Rillich et al., 2013; Knebel et al., 2017). Based on this robust motor 259 pattern, we measured the activity of trochanteral depressor MNs as representatives of the 
260 leg motor output, which participate, among others, in the stance phase during stepping.

\section{The DINs deliver the SEG descending control of the thoracic motor centers}

The SEG higher motor center commands are delivered downstream to the

264 peripheral motor center via a set of DINs (Kien, 1990a; b; Kien et al., 1990; Gal and

265 Libersat, 2006). We performed three experiments in which we stained one neck

266 connective, and found 146, 153, and again 153 stained neuronal somas in the SEG

267 (example: Fig. 1A). Therefore, assuming symmetry, and taking into account the few

268 neurons that send descending neurites in both connectives, there are almost 300 SEG

269 DINs (in accordance with Kien et al., 1990). To explore the anatomical relations between

270 the head ganglia DINs and the leg depressor MNs, we performed a double labeling of the

271 metathoracic N5a nerves and one neck connective. This revealed that many DIN

272 projections overlap with the arborization area of the N5a neurons, both ipsilaterally to the

273 stained connective and contralaterally (Fig, 1B and C). We could not determine whether

274 the DINs originate in the brain or the SEG, or whether they form synapses with the motor

275 neurons. However, the overlapping between DINs' projections and the leg motor neurons

276 are consistent with the previously reported functional connections, both for the SEG

277 DINs (cockroach: Gal and Libersat, 2008; locust: Kien, 1983; Kien and Altman, 1984)

278 and the brain DINs (cockroach: Bender et al., 2010; Mu and Ritzmann, 2008; Ridgel and

279 Ritzmann, 2005).

\section{The intact SEG fails to induce activity in the thoracic ganglia chain}

Previous studies have shown that headless insects, lacking both the supra- and 282 subesophageal ganglion, do not engage in spontaneous walking, and upon tactile 283 stimulation walk only briefly. However, when the SEG was left intact, the insects tended 284 to walk spontaneously and often uninhibitedly, without any additional stimulation (e.g. 285 stick insect: Bässler, 1983; locust: Kien, 1983; cockroach: Gal and Libersat, 2006). 286 Accordingly, several studies have also reported that without the SEG the leg CPGs are 287 inherently inactive in-vitro (Knebel et al., 2017 and references within). Therefore, we 
288 first explored whether the intact SEG induces spontaneous fictive leg motor activity in

289 the in-vitro thoracic ganglia chain preparations.

290 In all experiments $(\mathrm{N}=40)$ the pro- and mesothoracic ganglia depressor MN pools

291 were silent, whereas the meta-thoracic slow depressor was tonically firing (examples:

292 Fig. 1D and E before the stimulus; see also Knebel et al., 2017; Rillich et al., 2013). No

293 spontaneous motor bursts of action potentials were evident in any of the recordings.

\section{Stimulation of the SEG labial neuromere is sufficient for generating leg CPGs} activity in-vitro

Extracellular electrical stimulation of the SEG was previously reported to evoke bouts of walking in semi-intact locusts (Kien, 1990a). Following this report, we sought to was positioned into the SEG by way of a micromanipulator, and trains of short electrical pulses were applied, while the thoracic motor output was recorded. Due to the easy accessibility of the isolated preparation, we were able to direct the stimulating electrode to any of the three SEG neuropiles (the mandibular, maxillar, and labial neuromeres, from rostral to caudal). Furthermore, we were able to position the electrode in an area very close to the SEG longitudinal midline, and together with the high resistance small tip electrode used, the possible stimulation of the lateral tracts, in which most axons of brain DINs run directly to the ventral nerve cord, was limited.

Short electrical stimulations were delivered to 2-3 of the SEG neuromeres in four animals. Each trial consisted of 10 stimulations at intervals of $30 \mathrm{sec}$. In all experiments, a motor response in at least one thoracic ganglion was recorded. However, stimulation of

311 stimulations resulted in short responses (example of a mandibular stimulation: Fig. 1D;

312 overall medians: mandibular $1.4 \mathrm{sec}$ and maxillar $4.1 \mathrm{sec}$ ), whereas the labial stimulation

313 elicited prolonged activity with an overall median of $22.9 \mathrm{sec}$. Moreover, stimulation of

314 the labial neuromere elicited rhythmic bursting activity of up to six bursts, in 2-4 of the 315 recorded thoracic leg CPGs (example: Fig. 1E).

316 Rhythmic bursting activity in a sensory-deprived preparation is necessarily the 317 product of CPG activation. Our findings thus indicate that the labial neuromere of the 
318 SEG is sufficient to activate the leg CPGs in all three thoracic ganglia. Interestingly,

319 during all the in-vitro labial neuromere stimulations we observed prothoracic bilateral

320 synchronized excitation, whereas coupling patterns among the other recorded nerves

321 were varied (example: Fig. 1E).

The SEG has no effect on specific bursting properties of the leg CPGs

The muscarinic agonist pilocarpine is known to activate leg CPGs in isolated 324 nervous systems of arthropods (Chrachri and Clarac, 1987; Ryckebusch and Laurent, 325 1993, 1994; Ryckebusch et al., 1994; Büschges et al., 1995; Johnston and Levine, 2002; 326 Fuchs et al., 2011, 2012; Rillich et al., 2013; David et al., 2016). Recently, we 327 demonstrated that applying pilocarpine restrictively to each of the thoracic ganglia is 328 sufficient for inducing activity in all leg CPGs, including those of the untreated ganglia 329 (Knebel et al., 2017). To explore a tentative effect of the SEG on the CPG-CPG 330 interactions, we activated the thoracic CPGs by applying pilocarpine to the metathoracic 331 ganglion alone in an in-vitro preparation, and compared the motor output of the different 332 depressor MN pools both with the SEG intact and following its removal. Thus, while the 333 generation of rhythmicity arose from the caudal end of the ganglia chain, any SEG 334 modulatory influence had to be sent from the rostral end (pictogram: Fig. 2A).

335 Following pilocarpine application, rhythmic motor patterns were obtained in all 336 thoracic ganglia within 2-5 min (Fig. 2A). Before analyzing the coordination pattern 337 among the bursts of the different hemiganglia, we examined whether the SEG influences 338 the general properties of each CPG activity. To this end, we compared the spike 339 frequency in each recording, before and after SEG removal, and found no significant 340 change (Fig. 2B; N=31). We further examined the influence of the SEG on the CPGs 341 recruitment to the pharmacologically activated metathorcic CPGs; as only the 342 metathoracic ganglion was directly exposed to pilocarpine, all other CPG activity resulted 343 from activation of its CPGs. Therefore, the frequencies of the metathoracic CPGs and the 344 other CPGs should resemble each other (i.e. share a common frequency). Using the cross345 spectrum analysis, we tested whether the common frequencies differed with and without 346 the SEG (Fig. 2C). We found no effect of the SEG removal on the most common 347 frequencies in the meta-prothoracic and meta-mesothoracic bursting activity $(\mathrm{N}=31)$, and 
348 therefore concluded that the SEG does not affect the frequency-entrainment of the rostral

349 CPGs by the caudal metathoracic source of rhythmicity. Additionally, we found no SEG

350 effect on the shared frequencies between the two contralateral metathoracic CPGs.

351 Therefore, the SEG has no influence on the rhythmicity of the CPG network.

\section{The SEG modulates phase parameters among the CPGs}

As noted, we have recently reported that in in-vitro preparation, when pilocarpine was applied onto the metathoracic ganglion only, all the ganglia bilateral CPGs oscillate in antiphase while the ipsilateral CPGs are synchronized (Knebel et al., 2017). Here we

356 further explored whether the SEG is able to affect this coordination pattern. To this end, 357 we used the synchronization index to determine the synchrony level between each couple 358 of oscillators in our experiments (see Methods for details).

359 In line with our previous results, when the metathoracic ganglion was directly 360 activated with pilocarpine and the SEG was intact, all ipsilateral CPGs fired bursts of 361 action potentials in-phase. However, unlike in our previous report, in the presence of the 362 SEG the bilateral CPG also oscillated and fired in-phase (Fig. 3C). After removing the 363 SEG, while the ipsilateral CPGs oscillations remained synchronized, the activity of the 364 contralateral CPGs shifted towards anti-phase (Fig. 3D), similar to that recorded in our 365 previous report (where the SEG was removed at the dissection stage; Fig. 3E). Most 366 prominent was the change in the prothoracic ganglion CPGs, whose synchronization 367 index significantly dropped from 0.61 to -0.24 following SEG removal.

368 Taken together, the SEG synchronizes the activity of the two lateral sides of the 369 CPG network, without affecting the capacity for frequency entrainment and the burst 370 properties of the CPG network.

\section{Candidate SEG interneurons participate in the bilateral synchronization}

To explore the underlying neuronal circuitry behind the bilateral synchronizing 373 effect of the SEG, we simultaneously recorded the CPG motor outputs and the inter374 ganglia connectives. Based on multiple connective recordings (example: Fig. 2A) and 375 subsequent spike sorting, we were able to extract the activities of single SEG DINs. 376 Figure 4A-D presents an example of such SEG DIN activity. The cross-covariance 
analysis shows that this DIN activity was correlated with the firing of the two bilateral

378 MN pools in the prothoracic ganglion: that ipsilateral to the connectives recorded, and

379 that contralateral to them (Fig. 4B). The correlation of the DIN with the latter showed a

380 slight but consistent delay. This difference is visualized in the merged cross-covariance

381 shown in Fig. 4D, where the blue spot, representing a higher correlation between the ipsi-

382 and contralateral prothoracic MN pools in relation to the DIN firings, is smeared towards

383 the positive values of the contralateral prothoracic axis. Since the cross-covariance

384 between the DIN and the metathoracic CPG did not reveal a temporal relationship

385 between their activities (Fig. 4B, green line), this DIN activity is correlated to the activity

386 of specific CPGs.

387 By means of intracellular recordings we obtained further insights into the activity

388 of SEG interneurons during the metathoracic pilocarpine-induced rhythm. Figure 4E

389 presents an example of a SEG DIN recording. This cell's activity resembled that of the

390 DIN described above, as can be seen in the cross-covariance analysis: correlated activity

391 with both pro-thoracic MN pools, with a slight difference in the shape of the correlation

392 over time, and only weaker correlation with the metathoracic MNs (Fig. 4F). The

393 recording exhibits rather small action potentials and robust changes in the membrane

394 potential, indicating soma rather than axonal recordings. Hence, to correlate this DIN

395 activity with the prothoracic motor output, we used the values of the membrane potential

396 (and not only the spike on-times).

397 The two examples presented above demonstrate correlations between the output of 398 both of the prothoracic hemiganglia and an SEG DIN. However, in both cases, the left 399 and right CPGs were rather synchronized even irrespective of the DIN activity (Fig. 4C 400 and G), and it is impossible to distinguish whether the DINs' activity was connected to 401 one or both the prothoracic CPGs. Therefore, we further analyzed the findings from other 402 experiments, in which the CPG-GPG correlation was lower. Figure 4I presents an 403 example of an intracellular recording of an SEG interneuron in a preparation, in which 404 the prothoracic bilateral synchronization was relatively small (Fig. 4K). Nonetheless, 405 each of the prothoracic CPGs showed correlation with the SEG interneuron (Fig. 4J). 406 Moreover, the merged cross-covariance visualizes the small time window, in which the 407 SEG interneuron was correlated with both prothoracic CPGs (Fig. 4L), in contrast to the 
overall low bilateral correlation. These findings suggest that this SEG neuron interacts with both prothoracic MN pools. CPG network

413 such as the biogenic amine octopamine (e.g. Rand et al., 2012; Rillich et al., 2013). To

414 determine whether octopaminergic SEG neurons are centrally coupled to the leg CPGs

415 and are possibly involved in their synchronization, we aimed at specifically recording the 416 octopaminergic SEG DUM neurons.

417 Due to their unique bilateral descending neurites (Kien et al., 1990; Bräunig, 1991; 418 Cholewa and Pflüger, 2009), we were able to identify the DUM neurons' activity by 419 recording from both neck connectives, and from the pro-meso connective simultaneously 420 (see Methods for details; Fig. 5A; the different spike amplitudes seen in the left and right 421 connectives in this example is probably a result of different positions of the hook 422 electrode on the two connectives;). Repeating the cross-covariance analysis for this DUM 423 neuron and the two prothoracic CPGs indeed revealed a relatively strong correlation 424 among the activity of all three (Fig. 5B and D). In this example, again, the correlation of 425 each of the prothoracic CPGs with the DUM neuron exceeded the correlation between 426 both CPGs (Fig. 5C).

427 Additionally, we intracellularly recorded from a SEG DUM neuron as confirmed by 428 its labeling and action potentials (Fig. 6A): the neuron had a large soma of about $45 \mu \mathrm{m}$ 429 in diameter, was located medially on the dorsal posterior side of the SEG, and had a 430 symmetrical arborization pattern in the SEG, similar to those described by Bräunig 431 (1991; see laso Kien et al., 1990 and Bräunig and Burrows, 2004). Furthermore, its action 432 potentials were long lasting ( $3.5 \mathrm{~ms}$; Fig. 6B), and its soma was excitable (Fig. 6F), as 433 typical for DUM neurons (soma spikes: Heidel and Pflüger, 2006). Similar to the DUM 434 neuron identified by recording the connectives spikes (Fig. 5), this neuron's action 435 potentials showed a tendency to be synchronized with the output of both prothoracic 436 CPGs (The correlation was statistically significant only with the left nerve recording), 437 while the activity of the CPGs themselves was not correlated (Fig. 6C and D). 
In order to uncover the postsynaptic potentials (PSPs) that this neuron receives, we

439 hyperpolarized it for several minutes and thus minimized the action potentials generated.

440 We found that the DUM neuron received accurate and consistent information about co-

441 activation of the two prothoracic CPGs (example: Fig. 6G; analysis: Fig. 6H). Since the

442 DUM neuron EPSPs indicate synchrony between the bilateral CPGs, they might offer a

443 mean of coincidence detection. Interestingly, during the period of hyperpolarization, the

444 synchronization among the prothoracic CPGs noticeably increased (Fig. 6I).

445 Depolarization, on the other hand, did not evoke any immediate response (Fig. 6F).

\section{Discussion}

In this study we have demonstrated two major aspects of functional interactions between the higher locomotion centers of the SEG and the leg CPGs in the locust: (1) the

450 ability of the SEG to induce CPG activity, and (2) the role of the SEG in coordinating the 451 coupling among the leg CPGs. These interactions are mediated by the SEG DINs, 452 connecting the SEG with the thorax.

\section{The use of in-vitro preparations}

454 All the experiments presented here were conducted in-vitro. As previously 455 explained in Knebel et al. (2017), and as a common working hypothesis in CPG research 456 (e.g. Ayali and Lange, 2010 for review), it is advantageous to study the central interplay 457 of CPGs in the complete absence of sensory inputs. This reductionist approach is 458 consistent with the very definition of CPGs as a neuronal oscillator capable of performing 459 its tasks with no sensory regulation (Marder and Bucher, 2001). However, there are also 460 some clear limitations to this approach: namely, that conclusions, valid at the level of the 461 neuronal network and nervous system, may not be directly reflected in the actual 462 behavior, which is further shaped by additional sources, such as sensory inputs and the 463 animal's internal state, in the intact animal. Nonetheless, it is important to note that since 464 the central connections and interactions we studied here in-vitro are those underlying the 465 execution of the motor behavior, the insights gained in-vitro are instrumental for the 
generation of hypotheses and predictions regarding in-vivo insect locomotion.

\section{The SEG's ability to initiate different activity patterns in leg CPGs}

By means of electrical stimulation of SEG neurons we were able to activate each of the leg coxa-trochanteral CPGs. This finding, obtained in the isolated in-vitro preparation, indicates the sufficiency of the central connections between the SEG and the thoracic ganglia in activating the leg CPGs. Moreover, of the three SEG neuromeres, overall containing approximately 300 SEG DINs (Fig. 1A; see also Kien et al., 1990), we identified the labial neuromere as the most potent SEG area for CPG activation. Labial neuromere excitation induced prolonged bursting rhythms, similar to those obtained by activating the leg CPGs pharmacologically (compare examples in Fig. 1E and Fig. 2A; see also Knebel et al., 2017). These results are in accord with those reported by Kien (1983), as well as with our own preliminary experiments (not described herein), showing that stimulating the SEG in a semi-intact preparation results in leg movements (suppl. videos $1 \& 2$ ). Altogether, these findings suggest that the SEG is capable of inducing leg CPGs activity, and thus initiating stepping behavior. Moreover, they also suggest that by providing rhythmical excitatory and inhibitory signals to the leg CPGs, the SEG can maintain ongoing legged activity, as was previously suggested by Kien and Altman (1992). However, we also observed that intact connections to the unstimulated SEG were not sufficient by themselves to initiate spontaneous leg motor activity. It therefore seems that rather than constituting an autonomous "motivation center", the SEG functions as a relay station or a mediator, and depends on central inputs from the brain, from other CPGs, or on sensory information, in order to drive leg CPGs, (see also Kien and Altman, 1984). Moreover, the motor output induced by the SEG in-vitro, was monitored in all the leg CPGs but did not resemble any functional walking gait. This indicates that there is no complete "coordination program" or "walking motor program" conveyed by the SEG to

492 synchronization pattern of the leg CPGs was induced, suggesting that the SEG can 493 modulate the overall coordination of the locomotion network.

494 We have tried to limit the likelihood of stimulating severed axons, which originally 495 traveled from the brain to the SEG or thoracic ganglia, as explained in the methods and 
496 results sections. However, as with any extracellular stimulation, we cannot rule out the

497 possibility that undesired neurites were stimulated. Yet, our finding that the stimulation

498 of different neuromeres resulted in differentiated activation of the legs MN pools, and

499 that the coordination pattern established resembled that induced by the unstimulated

500 SEG, indicates that at least to the degree of affecting the leg motor output, stimulation of

501 brain DINs did not occur.

\section{The SEG bilateral synchronizing influence}

We characterized the role of the SEG in modulating the output of the leg CPGs network using an in-vitro preparation that included the thoracic ganglia and the SEG.

505 Based on our previous study (Knebel et al., 2017), we chose a specific paradigm of

506 restrictive pilocarpine application to the metathoracic ganglion. This allowed us to

507 investigate the output of the more rostral pro- and mesothoracic ganglia, their

508 interconnections, and their connection with the SEG, in the absence of any direct effects

509 of pilocarpine.

510 We found that the SEG does not alter the firing properties of any individual CPG,

511 nor their mutual bursting frequencies (Fig. 2B and C, respectively) but, rather, selectively

512 affects the phase relations of the bilateral CPG couples, most consistently those of the

513 prothoracic ganglion (Fig. 3). It is possible that the SEG can strengthen the inherent

514 synchronized bilateral coupling of the prothoracic CPGs (Knebel et al., 2017), and the

515 prothoracic ganglion, in turn, induces this coupling pattern upon its neighbors through the

516 synchronizing ipsilateral connections. However, from the neuronal network perspective,

517 since the ability of all the ganglia to induce activity in the CPGs of other ganglia and

518 influence their phase is equal (Knebel et al., 2017), it is more plausible that the SEG

519 modulates all bilateral inter-CPG connections, and that of the prothoracic ganglion is

520 simply the most responsive. Moreover, the SEG had no noticeable effect on coupling

521 among the ipsilateral CPGs, whose activities are synchronized independently of it. It

522 should be noted that these findings were obtained without the animal's brain, and future

523 studies will investigate its role in the CPGs coordination.

524 Supporting the findings described above, we found various examples of SEG cells

525 (Fig. 4-6) whose activities increased in phase with the synchronized output of the left and 
526 right prothoracic CPGs. In some of these examples, the correlations of the left and right

527 depressors with the SEG neurons were stronger than their mutual correlation (Fig. 4I-L,

528 5-6), suggesting that SEG neurons play an active role in bilateral synchronization.

529 However, due to the unexcitable properties of the soma membrane of those interneurons,

530 we were unable to elicit action potentials in most of our intracellular experiments, and

531 thus could not reach any causative conclusions regarding their role in the network.

532 It should be noted that due to our experimental procedure of restrictive 533 pilocarpine application, all correlated activity of SEG interneurons with the leg CPGs 534 must be the result of ascending information reaching the SEG and mirroring the CPG 535 activity. Such information can be referred to as "efference copy" (Jeannerod and Arbib, 536 2003), and is independent of sensory information (which was unavailable in our 537 experiments).

\section{The octopaminergic system interplay with the coordination of leg CPGs}

539 A potential candidate for the modulation of CPG-CPG interplay in insects is the 540 octopaminergic system (e.g. Rand et al., 2012; Rillich et al., 2013) and its prominent 541 members, the DUM neurons (for review: Libersat and Pflueger, 2004). Indeed, we found 542 that the activity of a descending SEG DUM neurons was correlated with the bilaterally 543 synchronized activity of the contralateral prothoracic CPGs (Fig. 5-6). This suggests 544 involvement of the octopaminergic system in the inter-CPG coordination, as all DUM 545 neurons in the SEG are octopaminergic (Bräunig, 1991; Stevenson and Sporhase546 Eichmann, 1995). Furthermore, we found that EPSPs recorded from a DUM neuron 547 reliably reflected the co-activation of the front leg depressors. Therefore, an ascending 548 neuron, or neurons, form synapses on the DUM neuron, eliciting changes in its 549 membrane potential correlated with the thoracic CPGs activity. This further confirms our 550 claim that a central efference copy of leg activity is delivered to the SEG.

551 Surprisingly, we found that upon silencing this DUM neuron, the prothoracic

552 bilateral synchronization increased. Taken together with the rest of our findings, this 553 result suggests that different neuronal elements in the SEG coordinate leg activity in a 554 complex, and not necessarily consistent, manner. 

walking

Walking behavior is dependent on the synergies of CPGs activities, sensory inputs, 558 and higher motor center regulation. Our results indicate the SEG as a potential integration 559 center: receiving descending inputs from the brain, as the anatomy of its neurons and 560 those of the brain suggests (Roth et al., 1994), and ascending information from both leg sensory organs (Kien and Altman, 1984) and the CPGs themselves, as presented here.

562 Since we did not observe in any of our in-vitro experiments fictive walking, 563 namely, a motor output that resembled a functional walking gait, we have to conclude 564 that, in the locust, functional inter-leg coordination during walking is only accomplished 565 by way of complementary sensory inputs or descending commands from the brain. This 566 was also reported for many other walking systems (Büschges et al., 2011) and vast 567 evidence suggests that sensory inputs plays a major role in walking coordination (e.g. 568 Borgmann et al., 2007, 2009; Daun-Gruhn, 2011; Fuchs et al., 2012).

We did, however, find that the SEG affects the overall leg CPG coordination.

570 Büschges et al. (1995; based on v. Holst, 1936) suggested that synchronized activity of 571 locomotive oscillators is the energetically cheapest way to couple them while ensuring 572 their joint frequency. As we have previously shown, the bilateral coupling among the 573 CPGs in the thoracic ganglia is flexible and rather weak compared to the ipsilateral 574 coupling (Knebel et al., 2017). It is therefore plausible that the level of bilateral 575 synchronization mediated by the SEG is sufficient to drive the two ipsilateral trios of 576 CPGs to oscillate at similar frequencies, as required for walking straight for example.

577 Previous studies have already suggested that the SEG is a bilateral mediator (Kien and 578 Altman, 1984; Kien et al., 1990; Bräunig and Burrows, 2004), but this was posited 579 mostly due to the anatomy of the majority of the DIN branches, which are contralateral to 580 their soma (Gal and Libersat, 2006). In light of this known DINs anatomy, and our 581 physiological findings, it is plausible that a decussation of information and commands 582 occurs in the SEG: ascending information about the CPGs activity and leg sensory inputs 583 is received ipsilaterally, while the DINs deliver commands downstream via the 584 contralateral descending axons, thus regulating the bilateral CPGs connections. 


\section{A comparison to vertebrate systems}

A comparison of the anatomy and function of the SEG and the vertebrate brainstem suggests some parallels, as previously suggested by Schoofs et al. (2014). In the

588 hindbrain-lesioned tadpole, for example, only short fictive swimming episodes could be

589 elicited in response to tactile stimulus (Li et al., 2006), similar to the short sequences of

590 walking that SEG-less insects perform (Gal and Libersat, 2006). Furthermore,

591 subpopulations of brainstem neurons mapped functionally and anatomically were shown

592 to be involved in locomotion initiation and halt (Shik et al., 1969; McClellan and

593 Grillner, 1984; Sirota et al., 2000; Cabelguen et al., 2003; Hägglund et al., 2010; Esposito

594 et al., 2014; Bouvier et al., 2015). Specifically, in all the different species examined,

595 stimulation of the mesencephalic locomotor region (MLR) induced locomotion-related

596 activity both in-vivo and in-vitro (Esposito and Arber, 2016). In the tadpole, neuronal

597 pacemakers in the hindbrain that send descending neurites to the swimming CPGs fire at

598 the beginning of each swimming cycle, presumably thereby maintaining a swimming

599 pattern (Soffe et al., 2009). Our experiments showed that the SEG can elicit several burst

600 cycles in the different leg depressors. It is therefore possible that, in a similar manner to

601 the tadpole hindbrain, repetitive activity of SEG DINs at the appropriate time during the

602 step cycle can excite the CPGs to be entrained in a functional manner.

603 Certain findings have also suggested that the brainstem mediates activity of both 604 sides of the vertebrate body, analogically to the role of the SEG reported here. Each of 605 the two-sided MLRs is capable of eliciting bilateral activation of the locomotion circuits 606 along the spinal cord by activating both sides of the reticulospinal tracts simultaneously, 607 thus delivering symmetrical commands to the spinal cord (Brocard et al., 2010).

608 Furthermore, it is within the medulla oblongata that the pyramidal tracts (which include 609 the motor corticospinal fibers), intersect to deliver information to the contralateral lower 610 motor neurons. Moreover, similar to the brainstem, out of which the cranial nerves 611 emerge, the SEG control the insect's mouthparts.

\section{Conclusion}

613 The SEG plays a regulative role in control of the arthropod legs. It has the ability to 614 induce movement in all legs in different coordination patterns by activating their CPGs, 
615 and it mediates their bilateral activity by adjusting the CPG coupling. Our findings have

616 revealed ways by which the SEG higher motor center serves as a higher motor center of

617 legged activity, as has been suggested in previous studies (Kien, 1983; Gal and Libersat,

618 2006; Bidaye et al., 2014). Overall, we show that the SEG interacts with the leg CPGs to

619 organize their joint activity; and, being situated in-between the brain and thorax, it

620 bridges between the brain centers, sensory inputs, and the motor circuits of the legs.

621 Interestingly, a comparison between the SEG and the vertebrate brain stem suggests some 622 parallels.

623 As noted above, findings based on in-vitro preparations are sometimes difficult to

624 interpret, especially when they do not completely correspond to the real behavior.

625 However, our findings did reveal functional connections between the leg CPGs and the

626 SEG. Hence, the importance of the results, for understanding walking behavior, is clear.

627 In light of the current findings and those of our previous study (Knebel et al., 2017), it is

628 now known that the locust CPGs, with and without the SEG inputs, are not naturally

629 coupled to produce any walking-like coordination. This is a consequence of the highly

630 modulated system that has to adjust to the heterogeneous environment in which walking

631 occurs, and therefore must avoid predetermined deterministic movements. Future work

632 should explore the effects and the roles of other mechanisms modulating the CPG

633 network, such as further higher motor centers and sensory inputs, and assess their

634 contribution to walking.

\section{References}

636 Ayali A, Lange AB. 2010. Rhythmic behaviour and pattern-generating circuits in the locust: key concepts and recent updates. J Insect Physiol [Internet] 56:834-843. Available from: http://www.ncbi.nlm.nih.gov/pubmed/20303972

Ayali A, Zilberstein Y. 2002. The locust frontal ganglion: a multi-tasked central pattern generator. Acta Biol Hung 55:129-135.

Bal T, Nagy F, Moulins M. 1988. The pyloric central pattern generator in Crustacea: a set of conditional neuronal oscillators. J Comp Physiol A 163:715-727.

Bässler U. 1983. Neural Basis of Elementary Behavior in Stick Insects. Berlin, Heidelberg: Springer Berlin Heidelberg. Available from: http://link.springer.com/10.1007/978-3-642-68813-3

Bässler U, Foth E, Breutel G. 1985. The inherent walking direction differs for the prothoracic and metathoracic legs of stick insects. J Exp Biol [Internet] 116:301311. Available from: http://jeb.biologists.org/content/116/1/301.short 
Bender JA, Pollack AJ, Ritzmann RE. 2010. Neural Activity in the Central Complex of the Insect Brain Is Linked to Locomotor Changes. Curr Biol [Internet] 20:921-926. Available from: http://www.ncbi.nlm.nih.gov/pubmed/20451382

Berkowitz A, Laurent G. 1996. Central generation of grooming motor patterns and interlimb coordination in locusts. J Neurosci 16:8079-8091.

Bidaye SS, Machacek C, Wu Y, Dickson BJ. 2014. Neuronal Control of Drosophila Walking Direction. Science [Internet] 344:97-101. Available from: http://www.sciencemag.org/cgi/doi/10.1126/science.1249964

Borgmann A, Hooper SL, Büschges A. 2009. Sensory feedback induced by front-leg stepping entrains the activity of central pattern generators in caudal segments of the stick insect walking system. J Neurosci [Internet] 29:2972-2983. Available from: http://www.ncbi.nlm.nih.gov/pubmed/19261892

Borgmann A, Scharstein H, Büschges A. 2007. Intersegmental coordination: influence of a single walking leg on the neighboring segments in the stick insect walking system. J Neurophysiol [Internet] 98:1685-1696. Available from: http://www.ncbi.nlm.nih.gov/pubmed/17596420

Bouvier J, Caggiano V, Leiras R, Caldeira V, Bellardita C, Balueva K, Fuchs A, Kiehn O. 2015. Descending Command Neurons in the Brainstem that Halt Locomotion. Cell [Internet] 163:1191-1203. Available from: http://dx.doi.org/10.1016/j.cell.2015.10.074

Bräunig P. 1991. Suboesophageal DUM Neurons Innervate the Principal Neuropiles of the Locust Brain. Philos Trans R Soc B Biol Sci [Internet] 332:221-240. Available from: http://rstb.royalsocietypublishing.org/cgi/doi/10.1098/rstb.1991.0051

Bräunig P, Burrows M. 2004. Projection patterns of posterior dorsal unpaired median neurons of the locust subesophageal ganglion. J Comp Neurol 478:164-175.

Brocard F, Ryczko D, Fenelon K, Hatem R, Gonzales D, Auclair F, Dubuc R. 2010. The transformation of a unilateral locomotor command into a symmetrical bilateral activation in the brainstem. J Neurosci [Internet] 30:523-533. Available from: http://www.ncbi.nlm.nih.gov/pubmed/20071515

Büschges A, Schmitz J, Bässler U. 1995. Rhythmic patterns in the thoracic nerve cord of the stick insect induced by pilocarpine. J Exp Biol [Internet] 198:435-456. Available from: http://www.ncbi.nlm.nih.gov/pubmed/9318078

Büschges A, Scholz H, El Manira A. 2011. New moves in motor control. Curr Biol [Internet] 21:R513-R524. Available from: http://www.ncbi.nlm.nih.gov/pubmed/21741590

Cabelguen J-M, Bourcier-Lucas C, Dubuc R. 2003. Bimodal locomotion elicited by electrical stimulation of the midbrain in the salamander Notophthalmus viridescens. J Neurosci 23:2434-2439.

Campbell JI. 1961. The anatomy of the nervous system of the mesothorax of Locusta migratoria migradorioides R. \& F. In: Proceedings of the Zoological Society of London. Vol. 137. Wiley Online Library. p 403-432.

Cholewa J, Pflüger H-J. 2009. Descending unpaired median neurons with bilaterally symmetrical axons in the suboesophageal ganglion of Manduca sexta larvae. Zoology (Jena) [Internet] 112:251-262. Available from: http://www.ncbi.nlm.nih.gov/pubmed/19423308

Chrachri A, Clarac F. 1987. Induction of rhythmic activity in motoneurons of crayfish 
695

696

697

698

699

700

701

702

703

704

705

706

707

708

709

710

711

712

713

714

715

716

717

718

719

720

721

722

723

724

725

726

727

728

729

730

731

732

733

734

735

736

737

738

739

740

thoracic ganglia by cholinergic agonists. Neurosci Lett 77:49-54.

Daun-Gruhn S. 2011. A mathematical modeling study of inter-segmental coordination during stick insect walking. J Comput Neurosci [Internet] 30:255-278. Available from: http://www.ncbi.nlm.nih.gov/pubmed/20567889

David I, Holmes P, Ayali A. 2016. Endogenous rhythm and pattern-generating circuit interactions in cockroach motor centres. Biol Open [Internet] 5:1229-1240. Available from: http://www.ncbi.nlm.nih.gov/pubmed/27422902

Dubuc R, Brocard F, Antri M, Fénelon K, Gariépy J-F, Smetana R, Ménard A, Le Ray D, Viana Di Prisco G, Pearlstein E, Sirota MG, Derjean D, St-Pierre M, Zielinski B, Auclair F, Veilleux D. 2008. Initiation of locomotion in lampreys. Brain Res Rev [Internet] 57:172-182. Available from: http://www.ncbi.nlm.nih.gov/pubmed/17916380

Esposito MS, Arber S. 2016. Motor Control: Illuminating an Enigmatic Midbrain Locomotor Center. Curr Biol [Internet] 26:R291-R293. Available from: http://dx.doi.org/10.1016/j.cub.2016.02.043

Esposito MS, Capelli P, Arber S. 2014. Brainstem nucleus MdV mediates skilled forelimb motor tasks. Nature [Internet] 508:351-356. Available from: http://www.ncbi.nlm.nih.gov/pubmed/24487621

Fritsch G, Hitzig E. 2009. Electric excitability of the cerebrum (Über die elektrische Erregbarkeit des Grosshirns). Epilepsy Behav [Internet] 15:123-130. Available from: http://linkinghub.elsevier.com/retrieve/pii/S1525505009001334

Fuchs E, Holmes P, David I, Ayali A. 2012. Proprioceptive feedback reinforces centrally generated stepping patterns in the cockroach. J Exp Biol [Internet] 215:1884-1891. Available from: http://www.ncbi.nlm.nih.gov/pubmed/22573767

Fuchs E, Holmes P, Kiemel T, Ayali A. 2011. Intersegmental coordination of cockroach locomotion: adaptive control of centrally coupled pattern generator circuits. Front Neural Circuits [Internet] 4:125. Available from: http://www.pubmedcentral.nih.gov/articlerender.fcgi?artid=3043608\&tool=pmcentr ez\&rendertype $=$ abstract

Gal R, Libersat F. 2006. New vistas on the initiation and maintenance of insect motor behaviors revealed by specific lesions of the head ganglia. J Comp Physiol A Neuroethol Sensory, Neural, Behav Physiol [Internet] 192:1003-1020. Available from: http://www.ncbi.nlm.nih.gov/pubmed/16733727

Gal R, Libersat F. 2008. A Parasitoid Wasp Manipulates the Drive for Walking of Its Cockroach Prey. Curr Biol [Internet] 18:877-882. Available from: http://www.pubmedcentral.nih.gov/articlerender.fcgi?artid=2850919\&tool=pmcentr ez\&rendertype $=$ abstract

Gordon IT, Whelan PJ. 2008. Brainstem modulation of locomotion in the neonatal mouse spinal cord. J Physiol [Internet] 586:2487-2497. Available from: http://doi.wiley.com/10.1113/jphysiol.2007.148320

Grabowska M, Godlewska E, Schmidt J, Daun-Gruhn S. 2012. Quadrupedal gaits in hexapod animals - inter-leg coordination in free-walking adult stick insects. J Exp Biol [Internet] 215:4255-4266. Available from: http://www.ncbi.nlm.nih.gov/pubmed/22972892

Gray JR, Robertson RM. 1998. Effects of heat stress on axonal conduction in the locust flight system. Comp Biochem Physiol Part A Mol Integr Physiol [Internet] 120:181- 
186. http://linkinghub.elsevier.com/retrieve/pii/S1095643398100284

Hägglund M, Borgius L, Dougherty KJ, Kiehn O. 2010. Activation of groups of excitatory neurons in the mammalian spinal cord or hindbrain evokes locomotion. Nat Neurosci [Internet] 13:246-52. Available from: http://www.ncbi.nlm.nih.gov/pubmed/20081850

Heidel E, Pflüger HJ. 2006. Ion currents and spiking properties of identified subtypes of locust octopaminergic dorsal unpaired median neurons. Eur J Neurosci 23:11891206.

v. Holst E. 1936. Über den „Magnet-Effekt“ als koordinierendes Prinzip im Rückenmark. Pflüger's Arch für die gesamte Physiol des Menschen und der Tiere [Internet] 237:655-682. Available from: http://link.springer.com/10.1007/BF01753051

Huber F. 1960. Untersuchungen über die Funktion des Zentralnervensystems und insbesondere des Gehirnes bei der Fortbewegung und der Lauterzeugung der Grillen. Z Vgl Physiol 44:60-132.

Hussaini SA, Menzel R. 2013. Mushroom Body Extrinsic Neurons in the Honeybee Brain Encode Cues and Contexts Differently. J Neurosci [Internet] 33:7154-7164. Available from: http://www.jneurosci.org/cgi/doi/10.1523/JNEUROSCI.133112.2013

Ito K, Shinomiya K, Ito M, Armstrong JD, Boyan G, Hartenstein V, Harzsch S, Heisenberg M, Homberg U, Jenett A, Keshishian H, Restifo LL, Roessler W, Simpson JH, Strausfeld NJ, Strauss R, Vosshall LB. 2014. A systematic nomenclature for the insect brain. Neuron 81:755-765.

Jeannerod M, Arbib M. 2003. Action monitoring and forward control of movements. In: Arbib M, editor. The Handbook of Brain Theory and Neural Networks. 2nd ed. Cambridge, MA: MIT Press. p 83-85.

Johnston RM, Levine RB. 2002. Thoracic leg motoneurons in the isolated CNS of adult Manduca produce patterned activity in response to pilocarpine, which is distinct from that produced in larvae. Invertebr Neurosci 4:175-192.

Kahn J, Roberts A. 1982a. The central nervous origin of the swimming motor pattern in embryos of Xenopus Laevis. J Exp Biol [Internet] 99:185-196. Available from: http://www.ncbi.nlm.nih.gov/pubmed/7130897

Kahn J, Roberts A. 1982b. Experiments on the Central Pattern Generator for Swimming in Amphibian Embryos. Philos Trans R Soc B Biol Sci [Internet] 296:229-243. Available from: http://rstb.royalsocietypublishing.org/cgi/doi/10.1098/rstb.1982.0004

Kaiser M, Libersat F. 2015. The role of the cerebral ganglia in the venom-induced behavioral manipulation of cockroaches stung by the parasitoid jewel wasp. J Exp Biol 218:1022-1027.

Kien J. 1983. The Initiation and Maintenance of Walking in the Locust: An Alternative to the Command Concept. Proc R Soc B Biol Sci [Internet] 219:137-174. Available from: http://rspb.royalsocietypublishing.org/cgi/doi/10.1098/rspb.1983.0065

Kien J. 1990a. Neuronal activity during spontaneous walking-I. Starting and stopping. Comp Biochem Physiol -- Part A Physiol [Internet] 95:607-621. Available from: http://www.sciencedirect.com/science/article/pii/030096299090747G

Kien J. 1990b. Neuronal activity during spontaneous walking--II. Correlation with 
stepping. Comp Biochem Physiol Part A [Internet] 95:623-638. Available from: http://www.ncbi.nlm.nih.gov/pubmed/1971548

Kien J, Altman JS. 1984. Descending interneurones from the brain and suboesophageal ganglia and their role in the control of locust behaviour. J Insect Physiol [Internet] 30:59-72. Available from: http://www.sciencedirect.com/science/article/pii/0022191084901082

Kien J, Altman JS. 1992. Preparation and execution of movement: parallels between insect and mammalian motor systems. Comp Biochem Physiol Comp Physiol [Internet] 103:15-24. Available from: http://www.ncbi.nlm.nih.gov/pubmed/1356693

Kien J, Fletcher WA, Altman JS, Ramirez JM, Roth U. 1990. Organisation of intersegmental interneurons in the suboesophageal ganglion of Schistocerca gregaria (Forksal) and Locusta migratoria migratorioides (Reiche \& Fairmaire) (Acrididae, Orthoptera. Int J Insect Morphol Embryol [Internet] 19:35-60. Available from: http://linkinghub.elsevier.com/retrieve/pii/0020732290900290

Kien J, Williams M. 1983. Morphology of Neurons in Locust Brain and Suboesphageal Ganglion Involved in Initiation and Maintenance of Walking. Proc R Soc B Biol Sci [Internet] 219:175-192. Available from: http://rspb.royalsocietypublishing.org/cgi/doi/10.1098/rspb.1983.0066

Knebel D, Ayali A, Pflueger H-J, Rillich J. 2017. Rigidity and Flexibility: The Central Basis of Inter-Leg Coordination in the Locust. Front Neural Circuits 10:112.

Li WC, Roberts A, Soffe SR. 2009. Locomotor rhythm maintenance: electrical coupling among premotor excitatory interneurons in the brainstem and spinal cord of young Xenopus tadpoles. J Physiol [Internet] 587:1677-1693. Available from: http://www.pubmedcentral.nih.gov/articlerender.fcgi?artid=2683956\&tool=pmcentr ez\&rendertype $=$ abstract

Li WC, Roberts A, Soffe SR. 2010. Specific brainstem neurons switch each other into pacemaker mode to drive movement by activating NMDA receptors. J Neurosci [Internet] 30:16609-16620. Available from: http://www.pubmedcentral.nih.gov/articlerender.fcgi?artid=3044868\&tool=pmcentr ez\&rendertype $=$ abstract

Li WC, Soffe SR, Wolf E, Roberts A. 2006. Persistent responses to brief stimuli: feedback excitation among brainstem neurons. J Neurosci [Internet] 26:4026-4035. Available from: http://www.ncbi.nlm.nih.gov/pubmed/16611819

Libersat F, Pflueger H-J. 2004. Monoamines and the Orchestration of Behavior. Bioscience 54:17-25.

Marder E. 2012. Neuromodulation of Neuronal Circuits: Back to the Future. Neuron [Internet] 76:1-11. Available from: http://dx.doi.org/10.1016/j.neuron.2012.09.010

Marder E, Bucher D. 2001. Central pattern generators and the control of rhythmic movements. Curr Biol [Internet] 11:R986-R996. Available from: http://www.ncbi.nlm.nih.gov/pubmed/11728329

Marder E, Bucher D. 2007. Understanding circuit dynamics using the stomatogastric nervous system of lobsters and crabs. Annu Rev Physiol 69:291-316.

McClellan AD, Grillner S. 1984. Activation of "fictive swimming" by electrical microstimulation of brainstem locomotor regions in an in vitro preparation of the lamprey central nervous system. Brain Res [Internet] 300:357-361. Available from: 
833

834

835

836

837

838

839

840

841

842

843

844

845

846

847

848

849

850

851

852

853

854

855

856

857

858

859

860

861

862

863

864

865

866

867

868

869

870

871

872

873

874

875

876

877

878

http://www.ncbi.nlm.nih.gov/pubmed/6733478

Miller WL, Sigvardt KA. 1998. Spectral analysis of oscillatory neural circuits. J Neurosci Methods 80:113-128.

Mu L, Ritzmann RE. 2008. Interaction between descending input and thoracic reflexes for joint coordination in cockroach: I. Descending influence on thoracic sensory reflexes. J Comp Physiol A Neuroethol Sensory, Neural, Behav Physiol [Internet] 194:283-298. Available from: http://www.ncbi.nlm.nih.gov/pubmed/18094976

Mulloney B, Smarandache C. 2010. Fifty Years of CPGs: Two Neuroethological Papers that Shaped the Course of Neuroscience. Front Behav Neurosci [Internet] 4:1-8. Available

from: http://www.pubmedcentral.nih.gov/articlerender.fcgi?artid=2917247\&tool=pmcentr ez\&rendertype $=$ abstract

Penfield W, Boldrey E. 1937. Somatic motor and sensory representation in the cerebral cortex of man as studied by electrical stimulation. Brain A J Neurol 60:389-443.

Pfeiffer K, Homberg U. 2014. Organization and functional roles of the central complex in the insect brain. Annu Rev Entomol [Internet] 59:165-84. Available from: http://www.ncbi.nlm.nih.gov/pubmed/24160424

Poeck B, Triphan T, Neuser K, Strauss R. 2008. Locomotor control by the central complex in Drosophila - An analysis of the tay bridge mutant. Dev Neurobiol [Internet] 68:1046-1058. Available from: http://www.ncbi.nlm.nih.gov/pubmed/18446784

Rand D, Knebel D, Ayali A. 2012. The effect of octopamine on the locust stomatogastric nervous system. Front Physiol [Internet] 3:288. Available from: http://www.pubmedcentral.nih.gov/articlerender.fcgi?artid=3429060\&tool=pmcentr ez\&rendertype $=$ abstract

Ridgel AL, Ritzmann RE. 2005. Effects of neck and circumoesophageal connective lesions on posture and locomotion in the cockroach. J Comp Physiol A 191:559573.

Rillich J, Stevenson PA, Pflüger H-J. 2013. Flight and Walking in Locusts-Cholinergic Co-Activation, Temporal Coupling and Its Modulation by Biogenic Amines. PLoS One [Internet] 8:e62899. Available from: http://www.pubmedcentral.nih.gov/articlerender.fcgi?artid=3650027\&tool=pmcentr ez\&rendertype $=$ abstract

Roth U, Kien J, Altman JS. 1994. Projections of suboesophageal descending interneurons in thoracic ganglia of the grasshopper Omocestus viridulus L. (Orthoptera?: Acrididae). Int J Insect Morphol Embryol [Internet] 23:275-291. Available from: http://www.sciencedirect.com/science/article/pii/0020732294900248

Rybak IA, Dougherty KJ, Shevtsova NA. 2015. Organization of the Mammalian Locomotor CPG: Review of Computational Model and Circuit Architectures Based on Genetically Identified Spinal Interneurons. eNeuro [Internet] 2. Available from: http://eneuro.sfn.org/cgi/doi/10.1523/ENEURO.0069-15.2015

Ryckebusch S, Laurent G. 1993. Rhythmic patterns evoked in locust leg motor neurons by the muscarinic agonist pilocarpine. J Neurophysiol [Internet] 69:1583-1595. Available from: http://www.ncbi.nlm.nih.gov/pubmed/8389831

Ryckebusch S, Laurent G. 1994. Interactions between segmental leg central pattern generators during fictive rhythms in the locust. J Neurophysiol [Internet] 72:2771- 
Ryckebusch S, Wehr M, Laurent G. 1994. Distinct rhythmic locomotor patterns can be generated by a simple adaptive neural circuit: Biology, simulation, and VLSI implementation. J Comput Neurosci [Internet] 1:339-358. Available from: http://www.ncbi.nlm.nih.gov/pubmed/8792239

Schmidt RA, Lee TD. 2005. Motor Control and Learning: A Behaviour Emphasis. 5th ed. Champaign, IL: Human kinetics.

Schoofs A, Hückesfeld S, Schlegel P, Miroschnikow A, Peters M, Zeymer M, Spieß R, Chiang AS, Pankratz MJ. 2014. Selection of Motor Programs for Suppressing Food Intake and Inducing Locomotion in the Drosophila Brain. PLoS Biol 12.

Shik ML, Severin F V, Orlovsky GN. 1969. Control of walking and running by means of electrical stimulation of the mesencephalon. Electroencephalogr Clin Neurophysiol [Internet] 26:549. Available from: http://www.ncbi.nlm.nih.gov/pubmed/4181500

Sigvardt KA, Miller WL. 1998. Analysis and modeling of the locomotor central pattern generator as a network of coupled oscillators. In: Annals of the New York Academy of Sciences. Vol. 860. . p 250-265.

Sirota MG, Di Prisco GV, Dubuc R. 2000. Stimulation of the mesencephalic locomotor region elicits controlled swimming in semi-intact lampreys. Eur J Neurosci 12:4081-4092.

Soffe SR, Roberts A, Li WC. 2009. Defining the excitatory neurons that drive the locomotor rhythm in a simple vertebrate: insights into the origin of reticulospinal control. J Physiol [Internet] 587:4829-4844. Available from: http://www.pubmedcentral.nih.gov/articlerender.fcgi?artid=2770150\&tool=pmcentr ez\&rendertype $=$ abstract

Stevenson PA, Kutsch W. 1987. A reconsideration of the central pattern generator concept for locust flight. J Comp Physiol A [Internet] 161:115-129. Available from: http://link.springer.com/10.1007/BF00609460

Stevenson PA, Sporhase-Eichmann U. 1995. Localization of octopaminergic neurons in insects. Comp Biochem Physiol B 11:203-215.

Strausfeld NJ, Hansen L, Li Y, Gomez RS, Ito K. 1998. Evolution, discovery, and interpretations of arthropod mushroom bodies. Learn Mem [Internet] 5:11-37. Available from: http://www.ncbi.nlm.nih.gov/pubmed/10454370

Strauss R. 2002. The central complex and the genetic dissection of locomotor behaviour. Curr Opin Neurobiol [Internet] 12:633-638. Available from: http://linkinghub.elsevier.com/retrieve/pii/S0959438802003859

Tastekin I, Riedl J, Schilling-Kurz V, Gomez-Marin A, Truman JW, Louis M. 2015. Role of the subesophageal zone in sensorimotor control of orientation in drosophila larva. Curr Biol [Internet] 25:1448-1460. Available from: http://dx.doi.org/10.1016/j.cub.2015.04.016

Wilson M. 1966. Insect Walking. Annu Rev Entomol [Internet]:103-122. Available from: http://www.annualreviews.org/doi/pdf/10.1146/annurev.en.11.010166.000535

921 Legends

\section{Figure 1}


922 SEG electrical stimulation elicits leg motor outputs. (A) Scan of the SEG after staining one neck connective. The dyed somas represent about half of the SEG DIN population. Scale bar: $100 \mu \mathrm{m}$ (B) Maximum intensity projection showing the double labeling of neck connective (red) and nerve 5A staining (green) in the metathoracic ganglion. Arrows in the pictograms and the scan indicate the stained nerves. Scale bar: $100 \mu \mathrm{m}$. (C) A single horizontal optical section from the framed zone in $(\mathbf{B})$, showing the overlapping area of the depressor motor neurons and DINs projections. Scale bar:100 $\mu \mathrm{m}$ (D) An example of a typical SEG stimulation of the neurons of the mandibular neuromere resulting in a brief elicited activity of the depressor MN of the pro- and mesothoracic ganglia. Scale bar: $5 \mathrm{~s}$ (E) An example of a typical SEG stimulation of the labial neuromere resulting in a prolonged rhythmic bursting activity of all recorded thoracic ganglia (Df, fast depressor; Ds, slow depressor; CI, common inhibitor). Scale bar: $5 \mathrm{~s}$.

\section{Figure 2}

935 Pharmacological stimulation of the metathoracic ganglion induces rhythmic bursting activity in all three thoracic ganglia. (A) The pictogram schematically presents the three thoracic ganglia and SEG. Pilocarpine was applied to the metathoracic ganglion only, as indicated by the gray filling. The arrows indicate the recording sites (4 depressor nerves and 3 connectives), as demonstrated by the recording trace. Scale bar:10 s. (B) The spike frequency throughout the $8 \mathrm{~min}$ of recording, for each hemiganglion (pro-, meso-, metathoracic hemigagnlia - T1, T2 and T3, respectively), with and without the SEG. No significant difference was found. (C) The most common frequency of the pro- and mesothoracic hemiganglia and the ipsilateral source of activation - the ipsilateral hemiganglia (T1 and T2, respectively), before and after SEG removal. Again, no significant difference was found. synchrony of the prothoracic CPGs before the SEG removal. The recording exemplifies the typical bursting activity of both hemiganglia. The circular histogram presents the phase of the prothoracic contralateral CPGs of all experiments. The bar filling colors represents the value of the synchronization index, as indicated by the color scale below. The sample size represents only the experiments in which we recorded the bilateral prothoracic activity. (B) As in (A) but for the activity of prothoracic CPGs after the SEG removal. The phase calculated for the two conditions was significantly different. Scale bar: 2 s. (C) A scheme of the CPGs phases before the SEG removal. The colors of the interconnecting lines between the pro-, meso- and metathoracic hemiganglia (upper, middle, and bottom circle pairs, respectively), represent the synchronization index values of each pair of CPGs, as shown on the color scale on the right. (D) As in (C) but after the SEG removal. (E) As in (C) but for experiments in which the SEG was removed from the thoracic ganglia chain at the dissection stage. Note that after the SEG removal, the bilateral CPG connections are drawn to anti-phase activity, while the ipsilateral connections remain in-phase, similarly to the experiments in which the SEG was not present from the beginning. $* * * \mathrm{p}<0.001$. 
SEG interneurons activity correlates with the prothoracic depressors joint activity. (A) Overlays of three simultaneous recordings from the connectives (one for each), indicated by the arrows in the pictogram. The black line represents the average vector of these overlays. The constant delay of $1.8 \mathrm{~ms}$ from the SEG downwards to the ipsilateral pro-meso connective indicates that the spike is generated by a neuron in the SEG, sending a descending neurite to all thoracic ganglia. Scale bar: $5 \mathrm{~ms}$ (B) Cross-covariance between the SEG DIN firing and the left and right prothoracic depressors (blue and red lines, respectively) and the right metathoracic depressor (green line). (C) Crosscovariance between the left and right prothoracic CPGs. (D) Merged cross-covariance of the left and right prothoracic depressors with the SEG DIN, from the narrow grayed window in (B). The intersection of the 0 values presents the time of the SEG DIN firing, and the colors indicate degree of correlation between the left and right depressors, as indicated by the color scale above. Note that the blue spot in (D) is smeared upwards, indicating that the peak of correlation is not at the spike onset, and a delayed prolonged elicited activity is recorded from the CPG contralateral to the DIN. Since no clear correlation is found between the DIN and the metathoracic depressor, as shown in (B), it is a candidate DIN for specifically synchronizing the prothoracic contralateral CPGs. (E) Simultaneous intracellular recording of a SEG neuron and the thoracic motor activity. Horizontal and vertical scale bars: $1 \mathrm{~s}$ and $10 \mathrm{mV}$, respectively. The confocal scan shows the recorded SEG neuron. Note that the neuron sends a neurite to the neck connective (indicated by the white arrow). Scale bar:80 $\mu \mathrm{m}$. (F) Cross-covariance between the SEG DIN membrane potential and the left and right prothoracic depressors (blue and red lines, respectively) and the right metathoracic depressor (green line). (G) As in (C), but for the prothoracic activity in the preparation shown in (E). (H) As in (D), but for the prothoracic and DIN activity in this preparation shown in (E) Note the resemblance to the SEG DIN shown in (D). (I) Simultaneous intracellular recording of SEG neuron and the thoracic motor activity. Horizontal and vertical scale bars: $1 \mathrm{~s}$ and $10 \mathrm{mV}$, respectively. The confocal scan shows the recorded SEG neuron. Scale bar: $80 \mu \mathrm{m}$. (J) Crosscovariance between the SEG interneuron spikes and the left and right prothoracic depressors (blue and red lines, respectively), and the right metathoracic depressor (green line). (K) As in (C), but for the prothoracic activity in the preparation shown in (I) (L) As in (D), but for the prothoracic and DIN activity in this preparation shown in (I). Note that despite the weak correlation between the left and right depressors, as seen in (K), the correlation at the time of the SEG neuron spike is clear, as seen in $(\mathbf{J})$.

\section{Figure 5}

A SEG DUM neuron activity is correlated with the prothoracic depressors joint activity. (A) An overlay of 3 recordings from the connectives, shown by the arrows in the pictogram. The almost simultaneous spike (constant delay of $0.4 \mathrm{~ms}$ ) in both the left and right connectives indicates that the spike is generated by a neuron in the SEG, sending two descending neurites to the thoracic ganglia, typical for SEG DUM neurons. 1006 Scale bar: $5 \mathrm{~ms}$. (B) Cross-covariance between the SEG DIN firing and the left and right prothoracic depressors (blue and red lines, respectively) and the right metathoracic 
1007 depressor (green line). (C) Cross-covariance between the left and right prothoracic CPGs. 1008 (D) Merged cross-covariance of the left and right prothoracic depressors with the SEG 1009 DIN, from the narrow grayed window in $(\mathbf{B})$. The intersection of the 0 values presents the 1010 time of the SEG DIN firing, and the colors indicate degree of correlation between the left 1011 and right depressors, as indicated by the color scale above.

\section{$1012 \quad$ Figure 6}

1013 A SEG DUM neuron activity is correlated with the prothoracic depressors joint 1014 activity. (A) Confocal scan shows the recorded SEG neuron. Scale bar: $80 \mu \mathrm{m}$. (B) 1015 Simultaneous intracellular recording of SEG neuron and the thoracic motor activity. 1016 Horizontal and vertical scale bars: $50 \mathrm{~ms}$ and $10 \mathrm{mV}$, respectively. Note the medial 1017 position of the neuron, its symmetrical arborization, and its long duration soma spikes. 1018 Together, these neuronal characteristics indicate that it is a SEG DUM neuron. (C) Cross1019 covariance between the SEG DIN firing and different sets of depressors (right 1020 prothoracic: blue; left prothoracic: red; right metathoracic: green). (D) Cross-covariance between the left and right prothoracic depressors (E) Merged cross-covariance of the left and right prothoracic depressors with the SEG DIN, from the narrow grayed window in (C). The intersection of the 0 values presents the time of the SEG DIN firing, and the colors indicate degree of correlation between the left and right depressors, as indicated by the color scale above. (F) Simultaneous intracellular recording of SEG neuron and the thoracic motor activity before, during, and after a short depolarization. Horizontal and vertical scale bars: $2 \mathrm{~s}$ and $10 \mathrm{mV}$, respectively. (G) Simultaneous intracellular recording of the SEG DIN and the thoracic motor activity during a permanent hyperpolarization. Horizontal and vertical scale bars: $2 \mathrm{~s}$ and $10 \mathrm{mV}$, respectively. (H), (I) and (J) are the same as (C), (D), \& (E) respectively, but for the SEG DIN during the permanent hyperpolarization, and conducted by analyzing the changes in its membrane potential. 


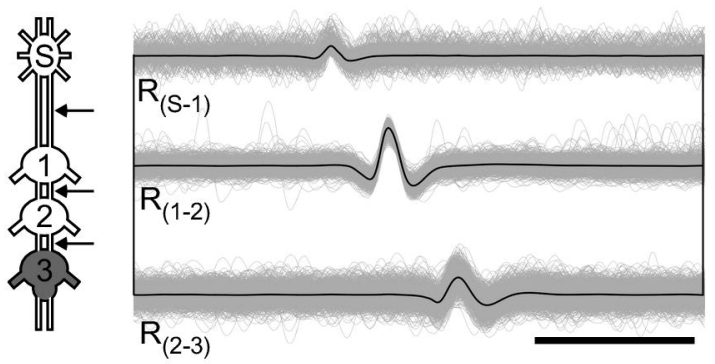

e

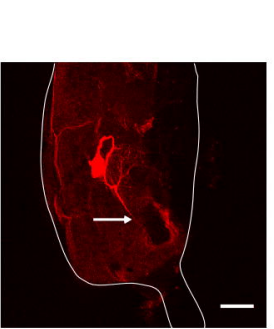

b$$
\text { c }
$$$$
\text { C }
$$

f

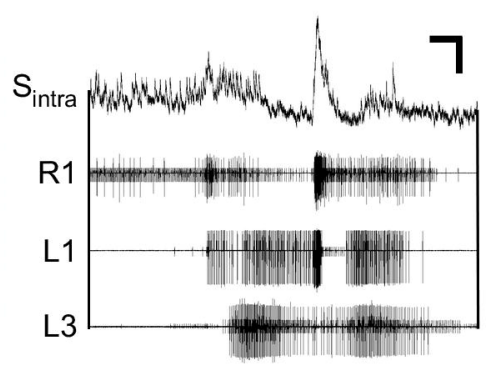

d

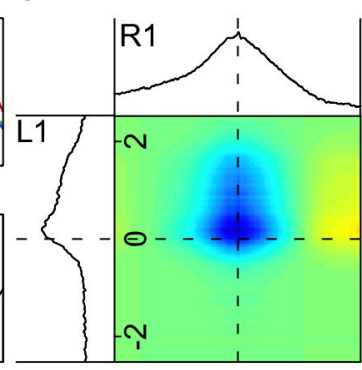

$\begin{array}{llll}\text { h } & -0.025 & 0 & 0.025\end{array}$

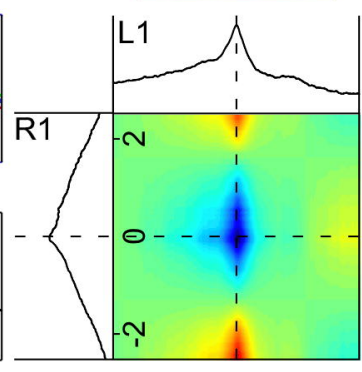

j
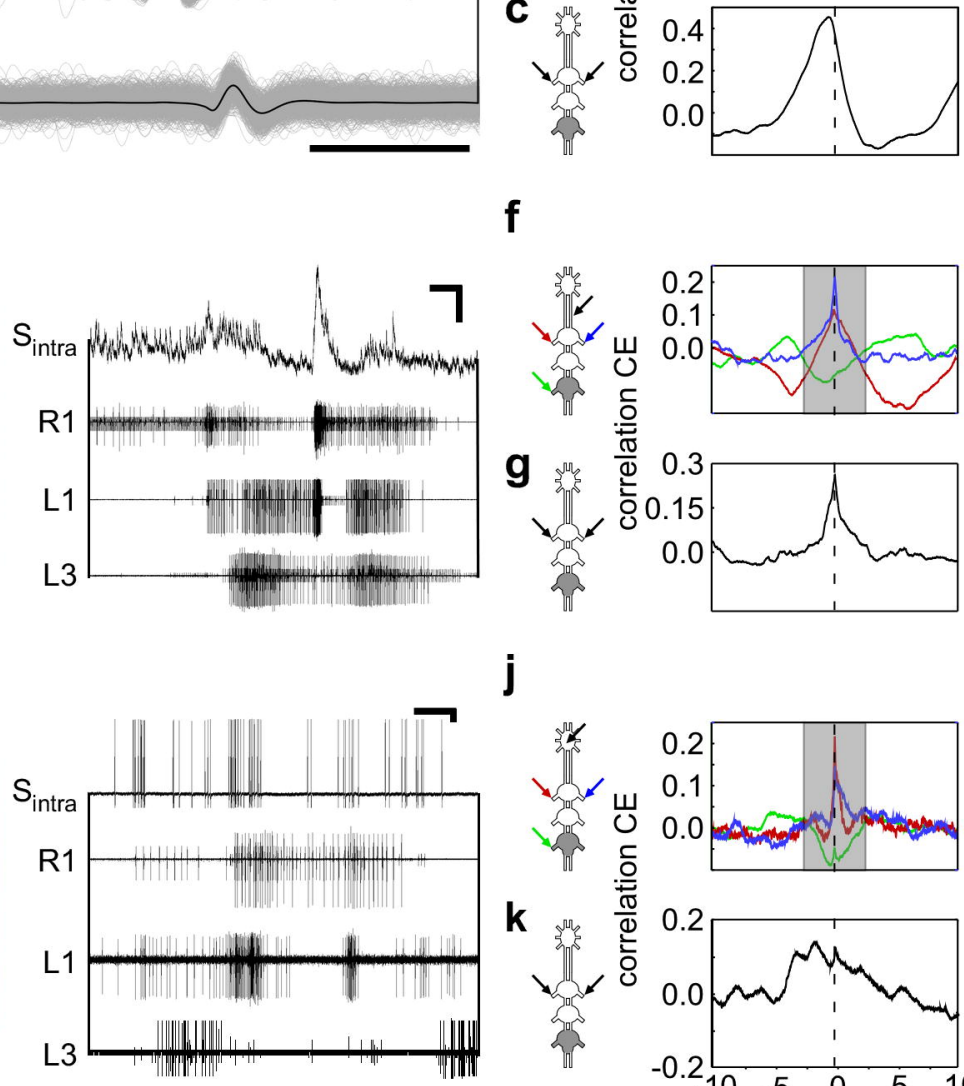

.

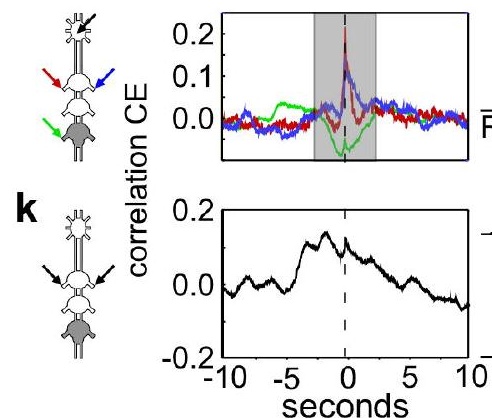

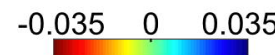

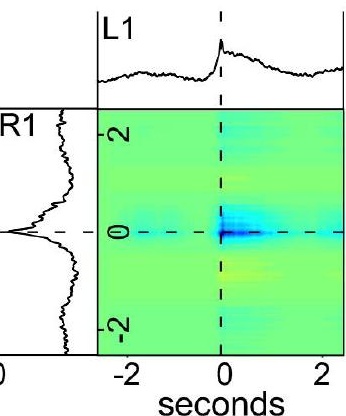



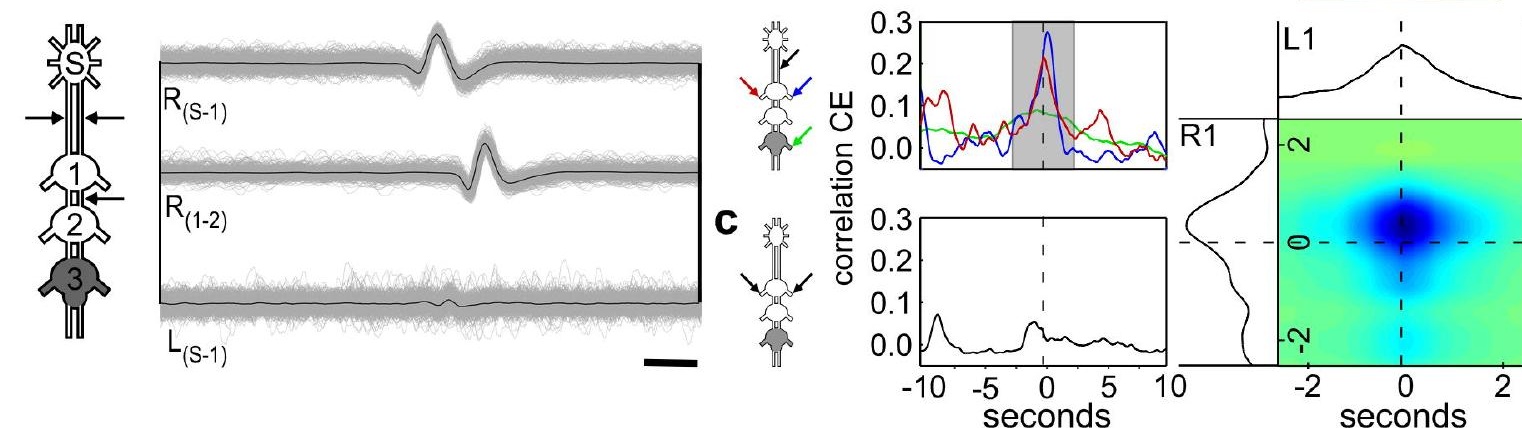


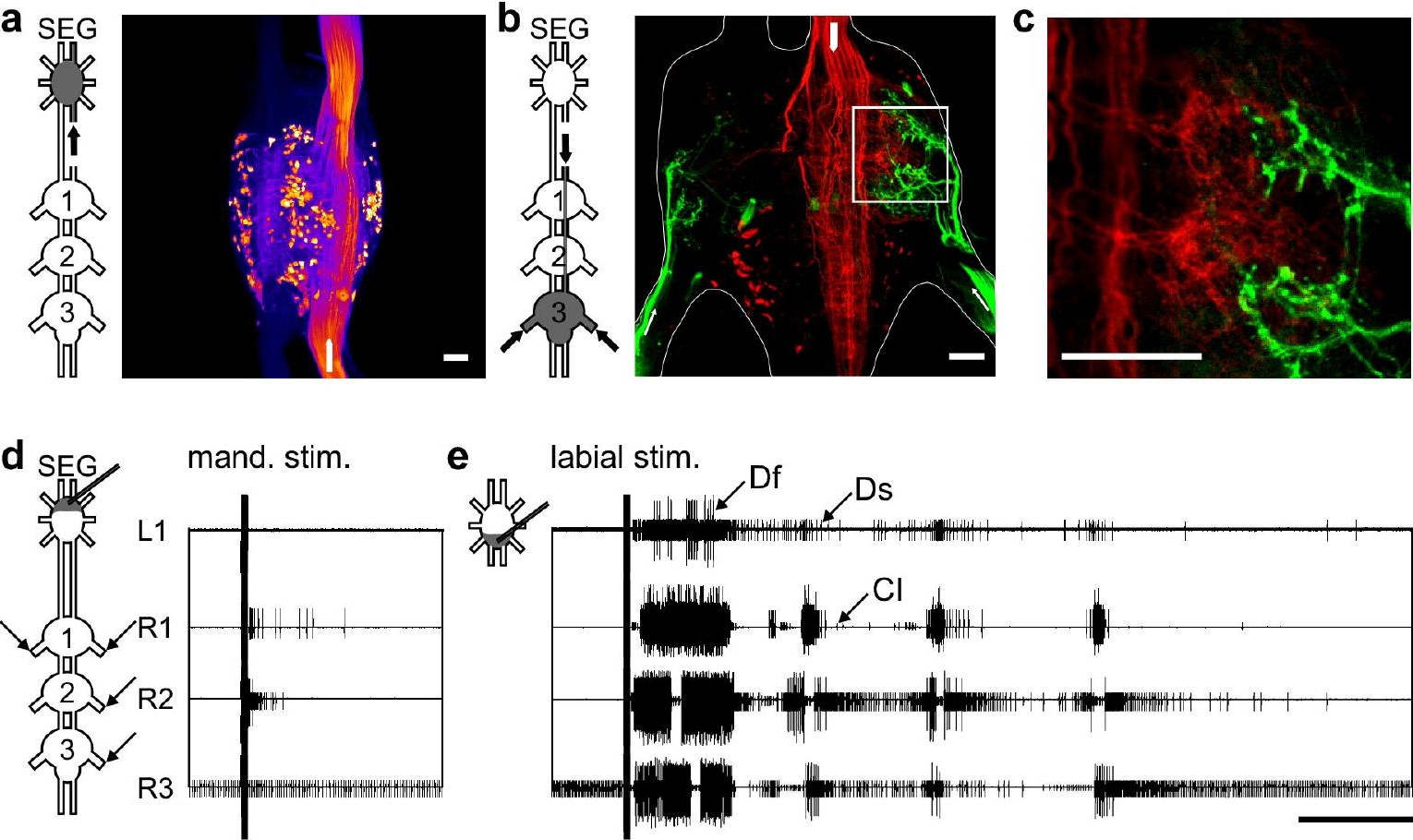


